This is the author's final, peer-reviewed manuscript as accepted for publication. The publisher-formatted version may be available through the publisher's web site or your institution's library.

\title{
Observation of public health risk behaviors, risk communication and hand hygiene at Kansas and Missouri petting zoos - 2010-2011
}

Gonzalo Erdozain, Katherine KuKanich, Benjamin Chapman, Douglas Powell

\section{How to cite this manuscript}

If you make reference to this version of the manuscript, use the following information:

Erdozain, G., KuKanich, K., Chapman, B., \& Powell, D. (2013). Observation of public health risk behaviors, risk communication and hand hygiene at Kansas and Missouri petting zoos - 2010-2011. Retrieved from http://krex.ksu.edu

\section{Published Version Information}

Citation: Erdozain, G., KuKanich, K., Chapman, B., \& Powell, D. (2013). Observation of public health risk behaviors, risk communication and hand hygiene at Kansas and Missouri petting zoos - 2010-2011. Zoonoses and Public Health, 60(4), 304-310.

Copyright: ( 2012 Blackwell Verlag GmbH

Digital Object Identifier (DOI): doi:10.1111/j.1863-2378.2012.01531.x

Publisher's Link:

http://onlinelibrary.wiley.com/doi/10.1111/j.1863-2378.2012.01531.x/full

This item was retrieved from the K-State Research Exchange (K-REx), the institutional repository of Kansas State University. K-REx is available at http://krex.ksu.edu 


\section{Corresponding Author:}

Dr. Douglas Powell

Professor, Food Safety

Department of Diagnostic Medicine/Pathobiology

Kansas State University

dpowell@ksu.edu

\section{Title:}

Observation of public health risk behaviors, risk communication and hand hygiene at Kansas and Missouri petting zoos - 2010-2011.

\section{Authors:}

Erdozain, Gonzalo; KuKanich, Katherine; Chapman, Benjamin; Powell, Douglas

Gonzalo Erdozain

College of Veterinary Medicine

Kansas State University

Manhattan, KS 66506

Katherine KuKanich

Assistant Professor

Department of Clinical Sciences

Kansas State University

Manhattan, KS 66506

Benjamin Chapman

Assistant Professor

Department of 4-H Youth Development and Family \& Consumer Sciences

North Carolina State University, NC Cooperative Extension

Raleigh, North Carolina

27616

Douglas Powell

Professor, Food Safety

Department of Diagnostic Medicine/Pathobiology

Kansas State University 
Manhattan, KS 66506

\section{Keywords:}

Petting zoo; zoonotic transmission; handwashing practices; risk behavior; animal contact

\section{Impacts}

- Effective handwashing is one tool to prevent spread of infection at petting zoos; however only $37 \%$ of observed visitors $(n=214 / 574)$ practiced hand hygiene upon exiting animal-contact areas in petting zoos in Kansas and Missouri.

- Visitors performed hand hygiene 4.8x more often when a staff member was present within, or at the exit to, the animal-contact area.

- Observed public health risk behaviors included: children (10/13 petting zoos) and adults ( $9 / 13$ petting zoos) touching hands to face within animal-contact areas; animals licking children's and adults' hands (7/13 and 4/13 petting zoos, respectively); and children and adults drinking within animal-contact areas (5/13 petting zoos each).

\section{Acknowledgements}

This study was supported by One Health Kansas (http:/ / onehealthkansas.kstate.edu) through an award from the Kansas Health Foundation to Kansas State University (PI: LC Freeman and BA Montelone). The authors acknowledge Katie Filion and Ramandeep Kaur for assistance in data collection.

\section{Tables' Legends}

Table 1: Hand hygiene station locations and supplies observed at 13 petting zoos' animal-contact area in Kansas and Missouri, U.S.

Table 2: Observed visitor behavior in a 30-minute period within 13 animal-contact areas at petting zoo events in Kansas and Missouri, U.S. 


\section{Observation of public health risk behaviors, risk communication and hand hygiene at Kansas and Missouri petting zoos - 2010-2011}

\section{Summary}

Outbreaks of human illness have been linked to visiting settings with animal contact throughout developed countries. This paper details an observational study of hand hygiene tool availability and recommendations; frequency of risky behavior; and, handwashing attempts by visitors in Kansas (9) and Missouri (4), U.S., petting zoos. Handwashing signs and hand hygiene stations were available at the exit of animal-contact areas in 10/13 and 8/13 petting zoos respectively. Risky behaviors were observed being performed at all petting zoos by at least one visitor. Frequently observed behaviors were: children (10/13 petting zoos) and adults ( $9 / 13$ petting zoos) touching hands to face within animal-contact areas; animals licking children's and adults' hands ( $7 / 13$ and 4/13 petting zoos, respectively); and children and adults drinking within animal-contact areas (5/13 petting zoos each). Of 574 visitors observed for hand hygiene when exiting animal-contact areas, 37\% ( $\mathrm{n}=214)$ of individuals attempted some type of hand hygiene, with male adults, female adults, and children attempting at similar rates (32\%, 40\%, and 37\% respectively). Visitors were $4.8 x$ more likely to wash their hands when a staff member was present within or at the exit to the animalcontact area $(136 / 231,59 \%)$ than when no staff member was present $(78 / 343$, $23 \% ; \mathrm{p}<0.001, \mathrm{OR}=4.863,95 \%$ C.I. $=3.380-6.998)$. Visitors at zoos with a fence as a partial barrier to human-animal contact were $2.3 x$ more likely to wash their hands $(188 / 460,40.9 \%)$ than visitors allowed to enter the animals' yard for contact $(26 / 114,22.8 \%$; $<<0.001, \mathrm{OR}=2.339,95 \% \mathrm{CI}=1.454-3.763)$. Inconsistencies existed in tool availability, signage, and supervision of animal-contact. Risk communication was poor, with few petting zoos outlining risks associated with animal-contact, or providing recommendations for precautions to be taken to reduce these risks.

\section{Introduction}

Outbreaks of zoonotic disease at petting zoos demonstrates that although contact with animals in public settings (such as fairs, petting zoos, and schools) can provide educational and entertainment opportunities (CDC, 2011) the potential to spread disease exists at these events if proper hygiene measures and precautions are not taken and reinforced. Human illness outbreaks have been linked to visiting petting zoos or similar settings with animal contact in the U.S., 
Canada, U.K., New Zealand, Australia, Ireland and the Netherlands (Erdozain and Powell, 2011). An October 2011 outbreak of E. coli O157:H7 at the North Carolina State Fair resulted in 25 illnesses; an August-September 2009, E. coli O157:H7 outbreak at Godstone Petting Farm in the U.K resulted in 93 illnesses (CDC, 2012; Griffin, 2010). The U.S. Centers for Disease Control and Prevention (CDC) has documented approximately 150 outbreaks of human infectious disease involving animals in public settings from 1996-2010 (CDC, 2011). Primary infectious agents of concern in these settings include Salmonella, Campylobacter, E. coli O157:H7 and Cryptosporidium (CDC, 2011). These infectious agents may be passed in animal feces and transmitted to humans via direct or indirect fecal-oral contact (Stirling et al., 2007). Children are at increased risk of infection in animalcontact settings due to certain factors and behaviors, including lack of awareness of the risk for disease, inadequate handwashing, lack of close supervision, and frequent hand-to-mouth activities (e.g., use of pacifiers, thumb-sucking, and eating) (CDC, 2011).

Petting zoos may restrict animal contact to touching or feeding animals over or through a fence, or permit people to enter animal enclosures while petting or feeding animals. These animal-contact settings may be present at state or county fairs, animal swap meets, pet stores, zoological institutions, circuses, carnivals, farm tours and educational exhibits at schools (Pike-Paris, 2006; Weese et al., 2007; Angulo et al., 2006). Animal species vary, although popular species in the U.S. include goats, sheep, rabbits, llamas, pigs, donkeys, calves, ducklings, chicks, reptiles, and rodents. Ruminants, such as cattle, goats, sheep and deer, can be sources of shiga-toxin producing E. coli, including E. coli O157:H7, and Cryptosporidium, while poultry, such as ducklings or chicks, may spread Salmonella spp. and Campylobacter.

Although regulations or guidelines for petting zoos exist, they vary by local and state health departments. Since 2000, both Pennsylvania (Act 211 of 2002) and North Carolina (Aedin's Law of 2005) have passed legislation for animal-contact venues (Marler, 2011). Both CDC and the U.K.'s Health Protection Agency (CDC, 2011; HPA, 2011) suggest handwashing is the most important prevention step for reducing disease transmission in animal-contact settings.

Hand hygiene compliance rates at petting zoos are highly variable, often averaging below 50\%, and are dependent on venue layout (McMillan et al., 2007; 
Weese et al., 2007; Anderson and Weese, 2011). Weese et al. (2007) observed hand hygiene compliance by petting zoo visitors at 36 venues in Ontario, Canada. Results indicated hand hygiene compliance rates by visitors at petting zoos varied between $0-77 \%$ (mean value $30.9 \%$ ), and the authors suggested factors associated with increased compliance was availability of hand hygiene stations near the exit, running water, and hand hygiene signage (Weese et al., 2007). A follow-up study by Anderson and Weese (2011) in Ontario, Canada, found 58\% of visitors performed some form of hand hygiene (either using water, soap and water, or hand sanitizer), and two interventions (improved signage while offering hand sanitizer, and verbal hand hygiene reminders by venue staff) were associated with increased hand hygiene compliance (Anderson and Weese, 2011). U.K. health officials currently recommend handwashing stations with soap and water only (no wipes or sanitizers).

While some studies suggest inadequate handwashing facilities may have contributed to enteric disease outbreaks (Bender and Shulman, 2004), or washing hands was protective against illness (Friedman et al., 1998; Smith et al., 2004; CDC, 2011), others suggest relevant infectious agents may be aerosolized and inhaled (Varma et al., 2003), thus not prevented with handwashing. Handwashing tool selection may also contribute to the success of hand hygiene as a preventive measure, as some outbreak investigations have reported alcoholbased hand sanitizer was not protective against illness, especially when hands are soiled (CDC, 2005-1; Goode et al., 2009).

The objective of this study was to characterize public health risk behavior and hand hygiene practices by visitors at Kansas and Missouri petting zoos. Secondary objectives included determining the presence of risk communication tools and hygiene station availability in these zoos.

\section{Methodology}

A convenience sample of 13 public events advertizing "petting zoo" in Kansas $(n=9)$ and Missouri $(n=4)$ was used for this study. The study was given exempt status from the Institutional Review Board at the authors' university due to observation of public behavior. All observations were anonymous and no personal information was collected. Petting zoo events were identified through Internet searches and word-of-mouth. Unannounced visits were performed during the autumn of 2010 and summer of 2011. Public petting zoo attractions 
were located at both temporary and permanent events, including zoos, farms, and pumpkin patches. Information collected included zoo duration (permanent vs. temporary), animal species, types of animal contact permitted, handwashing facilities, signage, hand hygiene, and risk behaviors. Hand hygiene station location was noted in relationship to animal enclosures. Observations were conducted either inside or outside of animal-contact areas, depending on the size and nature of the petting zoo. While conducting behavioral observations, observers were positioned such that visitor actions were in plain view. Behavioral observations were conducted during 30-minute, randomly selected periods in each petting zoo throughout the day. During behavioral observation periods, data were collected on visitor actions deemed to be an infection risk (e.g. eating animal food, animals licking a child's face) within animal-contact areas. Number of visitors observed performing each behavior in each zoo during allotted time periods was recorded; total number of visitors in each zoo during each allotted period was not collected due to limited observers and high volume of visitors entering and exiting each zoo. Hand hygiene behaviors of visitors within animal-contact areas were observed for 30 minutes per petting zoo visit, and information collected included gender, age (adult, child), hand hygiene attempt upon exiting the animal-contact area (yes or no), and materials used (sanitizer; soap and water). The number of people exiting an animal-contact area who made an attempt at hand hygiene was recorded and compared with total number of people exiting that area within that 30-minute period. Hand hygiene performed within the animal-contact area (where available) was not recorded, as further animal contact may have occurred prior to exit. The materials used for each hand hygiene attempt were recorded, but hand hygiene technique was not evaluated.

Statistical analysis - Descriptive statistics were used for summarizing risk behaviors, signage, and hand hygiene tools. Fisher's exact test was used to test whether a risk factor observed in an animal-contact area was associated with staff presence or specific signage discouraging that behavior. Chi-square analysis was used to analyze whether hand hygiene attempts were different among adults and children, or between adult male and female visitors. Similarly, chisquare analysis was used to determine whether hand hygiene performance was associated with visitors being allowed animal contact by entering animal yards or associated with some form of enclosure such as a fence. Chi-square analysis was also used to determine if an association was present between hand hygiene 
performance and presence of a staff member. Odds ratios with $95 \%$ confidence intervals were calculated when statistical associations were significant. Significance was set at $\mathrm{p}<0.05$ for all comparisons.

\section{Results}

Animals most commonly present in petting zoos included goats, deer, chicks, ducks, sheep, and cows. Goat kids were particularly popular among petting zoos $(n=11 / 13)$, including $6 / 7$ petting zoos that permitted visitors to enter animal enclosures. Additionally, one petting zoo allowed children to enter, pet and sit among tortoises. Seven out of 13 petting zoos permitted visitors to enter animal enclosures, while the remaining six petting zoos restricted petting/feeding to be done over or through a fence. Twelve petting zoos sold or provided free animal food for direct animal feeding by visitors.

Hand Hygiene - Hand hygiene facilities were available at the exit of 7/13 petting zoos' animal-contact areas. The remaining six petting zoos had hand hygiene facilities within or near animal-contact areas. Not all facilities were considered adequate. One petting zoo had a sink without soap, and no sanitizer was available. At another petting zoo, 2/3 sanitizer dispensers were empty and there was no accessible sink or soap for handwashing. A summary of hand hygiene station locations and supplies at animal-contact areas is provided in Table 1.

\section{INSERT TABLE 1 HERE}

Eating Areas and Restrooms - Separate eating areas (away from animal enclosures) were noted in $11 / 13$ petting zoos. All observed petting zoos had restrooms available to visitors, and all restrooms were deemed to be in good working condition. Soap, water and paper towels were available in the restrooms at 12/13 petting zoos. Three petting zoos provided instructions for handwashing/sanitizing in restrooms.

Signage - Signs to encourage handwashing were available at petting zoos in the following locations: exit $(n=10 / 13)$ of animal-contact areas and inside restrooms $(n=3 / 13)$, but signs were not noted at the main zoo entrance or entrance to eating areas at any visited events. Directions to the location of handwashing stations were provided on signs in four petting zoos. Signs gave instructions to wash hands prior to eating, drinking or smoking $(n=3 / 13)$, prior to touching animals $(n=1 / 13)$, after touching animals $(n=12 / 13)$, after handling animal food $(n=4 / 13)$, 
and after using the restroom $(n=3 / 13)$. Where signs included guidelines for how to wash hands $(n=1 / 13)$, details included: use soap and running water, and rub hands vigorously for 15 seconds. Other signage near animal-contact areas included instructions: not to feed animals while eating/drinking/smoking $(n=4 / 13)$; to wash hands prior to touching face $(n=1 / 13)$; not to eat animal food $(\mathrm{n}=1 / 14)$; not to eat or drink human food in animal-contact areas $(\mathrm{n}=6 / 13)$; and to cover open wounds $(n=1 / 13)$.

Staff and Animal-contact Area Cleanliness - In six petting zoos $(\mathrm{n}=6 / 13)$ a staff member was present monitoring activity in the animal-contact area; in some petting zoos, a staff member was seen cleaning the yard. In one petting zoo, goat kids were observed escaping through the fenced enclosure and roaming freely; no staff member was present. All petting zoos observed were deemed to have clean animals (animals appeared well kept and healthy). At petting zoos where visitors were allowed to enter the animal-contact area $(n=7 / 13)$, small amounts of urine and feces were observed in 5/7 enclosures. No staff member was observed verbally encouraging hand hygiene or discouraging risk-behaviors.

Visitor Behaviors - A summary of observed visitor behavior in petting zoos can be found in Table 2. In total, 496 behavioral observations at 13 petting zoos were made. The most commonly recorded behaviors were visitors touching their hands to their faces and animals licking visitors' hands. No association was identified between hand-touching-face behavior by children $(\mathrm{p}=0.563)$ or adults $(\mathrm{p}=0.216)$, and presence of a staff member within the animal-contact area; a sign discouraging this behavior was present in $1 / 13$ petting zoos. No association was made between animals licking children's hands $(p=0.286)$ nor animals licking children's faces $(\mathrm{p}=0.192)$, and presence of a staff member. However, animals were observed to lick children's hands in $2 / 6$ petting zoos with a staff member present and $5 / 7$ petting zoos without a staff member present. No signs were present in any petting zoo discouraging visitors from allowing animals to lick their hands, but 12/13 petting zoos provided food for visitors to feed animals. Children eating $(p=1.000)$ or drinking $(p=0.265)$ within animal-contact areas was not associated with staff presence, nor were these behaviors associated with signs discouraging them. In one petting zoo, three children were observed picking up animal feces. This site did not have a staff member present, a sign discouraging touching feces, nor available handwashing facilities at the exit of the animal-contact area. The petting zoo where one child was found consuming 
animal food in an animal-contact area did not have a staff member present nor a sign discouraging this behavior.

\section{INSERT TABLE 2 HERE}

Visitor Hand Hygiene - Data for hand hygiene behavior of visitors were recorded from seven petting zoos with hand hygiene facilities at the exit to the animalcontact areas. The hand hygiene behavior of 574 individuals was observed. Adults accounted for $55 \%(n=316)$ of observations, consisting of $40 \%$ males $(n=126)$ and $60 \%$ females $(n=190)$. Children accounted for $45 \%(n=258)$ of observations. A total of 214/574 individuals (37\%) made a hand hygiene attempt while exiting the animal-contact area during observation periods. No difference in attempt percentage was noted between adults $(n=118 / 316)$ and children $(n=96 / 258)(p=1.000)$ or between adult males $(n=41 / 126)$ and adult females $(\mathrm{n}=77 / 113)(\mathrm{p}=0.156)$. Visitors performed hand hygiene more often when a staff member was present within or at the exit to the animal-contact area $(n=136 / 231$, $59 \%)$ than when no staff was present in these areas $(\mathrm{n}=78 / 343,23 \%)(\mathrm{p}<0.001$, $\mathrm{OR}=4.863,95 \%$ C.I. $=3.380-6.998)$. Visitors in petting zoos where animal contact occurred over or through a fence performed hand hygiene more often $(\mathrm{n}=188 / 460,40.9 \%)$ than visitors in petting zoos who were allowed to enter an animals' yard for contact $(\mathrm{n}=26 / 114,22.8 \%)(\mathrm{p}<0.001, \mathrm{OR}=2.339,95 \% \mathrm{CI}=1.454-$ 3.763).

Limitations - Handwashing compliance can be affected by factors that were not assessed in this study, such as peer pressure and layout of the animal-contact area and handwashing stations. Additional research should be undertaken to observe how each affects compliance. A second limitation was an unequal number of visitors observed at each petting zoo. Another limitation was the inability to record the total number of visitors per petting zoo during risk behavior observation periods, which would have allowed for statistical analysis at the individual level rather than petting zoo. Finally, this report represents observations from Kansas and Missouri petting zoos, and while these petting zoos had characteristics of petting zoos throughout the U.S., results may vary based on geographical location.

\section{Discussion}


Seven out of 13 petting zoos allowed visitors to enter the animals' yards, which may increase opportunity for certain risk behaviors (increased contact with animals and exposure to feces and other waste) and exposure to infectious organisms. Disease transmission may occur through direct contact with animals or animals' waste, as was the case with a North Carolina outbreak which affected 108 people (CDC, 2005-1); indirectly through contact with surroundings (such as fencing, Griffin, 2010); or even aerosolization as observed in an E. coli O157:H7 outbreak at a county fair in Oregon, in which 60 people fell ill (CDC, 2005-2). Small amounts of urine and feces were observed in 5/7 petting zoos where visitors were allowed to enter animal yards. This may be of concern with young children, specifically toddlers, who may fall down frequently (Moreno, 2009), when visitors are permitted to bring items such as bottles, pacifiers, food or toys into animal enclosures, or for immunosuppressed individuals. Animal hygiene was observed in this study, and all visited zoos were deemed to have clean animals (animals appeared well kept and healthy). Although this was a positive finding, it may provide little reassurance, as animals infected with enteric pathogens may show no signs of illness, and pathogens may be shed intermittently (McMillan et al., 2007). Additionally, animals considered to be of a particularly high risk of disease shedding generally include young ruminants, young poultry, reptiles, amphibians, and ill animals (NASPHV, 2005). In this study, young ruminants, young poultry and reptiles were among animals encountered at zoos. Goat kids were particularly popular among petting zoos (11/13), including $6 / 7$ zoos that permitted visitors to enter animal enclosures. Additionally, one petting zoo allowed children to enter, pet and sit among tortoises. CDC's Animal-Specific Guidelines recommends against this type of exposure due to risk of acquiring Salmonella from reptiles: "Do not keep reptiles (turtles, snakes, lizards) in facilities with children aged $<5$ years, nor should children aged $<5$ years be allowed to have direct contact with these animals"(CDC, 2011; CDC, 2010).

Public health risk behaviors were observed being performed by at least one visitor in all $(n=13)$ petting zoos visited. These behaviors may put visitors at risk of acquiring a zoonotic disease. Although touching hands to face within the animal-contact area was the most frequently noted behavior, one child was observed picking up animal feces, and one child was seen eating animal food. Frequent hand-to-mouth behavior, such as sucking on pacifiers, eating, or drinking within animal-contact areas, has been shown to increase the risk of $E$. 
coli acquisition in children (Moreno, 2009). In 2004-2005, two separate outbreaks of E. coli O157:H7, located in North Carolina and Florida, resulted in over 187 combined illnesses. In both outbreaks, extensive direct animal contact and behaviors such as falling or sitting on the ground or using a "sippy" cup within enclosures were associated with illness (CDC, 2005-1). During an outbreak of $E$. coli O157:H7 at the Western Fair in Ontario, Canada, it was noted that animal feed provided to visitors in edible ice-cream cones may have contributed to disease transmission (Warshawsky et al., 2002). Risk communication tools and hygiene stations are essential to inform visitors of potential risks and facilitate compliance of public health recommendations. Both varied greatly in Kansas and Missouri petting zoos.

While most permanent petting zoos (9/11) had soap and water available, not all petting zoos (7/13) had paper towels and some (mostly temporary petting zoos) had only hand sanitizer. Yamamoto et al. (2005) found paper towels to be more effective for removing bacteria from fingertips than palms and fingers; paper towels are also less likely to spread bacteria and contaminate the surroundings when compared with hot air driers or jet air driers (Redway, 2008). Petting and feeding animals allows for organic material to contaminate hands, lowering efficacy of alcohol-based hand sanitizers against pathogens. CDC (2011) and HPA (2011) recommend handwashing with water and soap as the best method of hand hygiene. CDC categorizes alcohol-based hand sanitizers as a handsanitizing agent, but recommends these be used only when soap and water are not available, as sanitizers are less effective on visibly soiled hands.

Overall hand hygiene compliance upon exiting animal-contact areas was poor in this study ( $\mathrm{n}=214 / 574,37 \%)$, and consistent with findings $(30.9 \%)$ by Weese et al. (2007) at Ontario petting zoos. Increased handwashing compliance with the presence of a staff member, suggests that the oversight has a direct, positive impact on visitors' hand hygiene compliance. An individual's behavior has been shown to change when he or she is aware of being watched (Gould et al., 2007). Similarly, peer pressure has been reported to improve hand hygiene (World Health Organization, 2009; Wilson et al., 2011). Visitors in petting zoos where animal contact occurred over or through a fence also performed hand hygiene more often than visitors in petting zoos who were allowed to enter an animals' yard for contact, which may have been impacted by availability or location of hygiene stations, presence or location of signs, or additional perception of risk 
factors that may have influenced handwashing behavior but not assessed in this study. Other factors that may influence hand hygiene compliance include understanding of public health risks involved with animal-contact, and understanding of the benefits of handwashing in minimizing these risks. More research is needed in this area to understand how these factors relate to each other and influence hand hygiene compliance to identify targets where behavior changing interventions and methods could be applied.

While the presence of a staff member appears to increase hand hygiene compliance, it appeared to have no effect on reducing risky behaviors. While educational signs have the potential to convey risks and encourage hand hygiene (Chapman et al., 2010), no effect was seen in this study and further research into ideal content and location of signage in petting zoos may be warranted. Building on Anderson and Weese's (2011) findings, this study supports the importance of amending current best practice guidance to explicitly include suggestions that a staff member be located in animal contact areas to passively and actively encourage hand hygiene as well as risk-reduction behavior. 


\section{References}

1. Anderson, M.E., Weese, J.S. 2011. Video observation of hand hygiene practices at a petting zoo and the impact of hand hygiene interventions. Epidemiol Infect 4:1-9.

2. Angulo, F., Bender, J., Demma, L., Eidson, M., J., Steinmuller, N. 2006. Outbreaks of enteric disease associated with animal contact: Not just a foodborne problem anymore. Clin Infect Dis 43:1596-1602.

3. Bender, J. B., S. A. Shulman. 2004. Reports of zoonotic disease outbreaks associated with animal exhibits and availability of recommendations for preventing zoonotic disease transmission from animals to people in such settings. J Am Vet Med Assoc 224(7): 1105-1109.

4. Centers for Disease Control and Prevention. Outbreaks of Escherichia coli O157:H7 associated with petting zoos - North Carolina, Florida, and Arizona, 2004 and 2005. MMWR 2005;54(No.50):[1-4].

5. Centers for Disease Control and Prevention. Compendium of measures to prevent disease associated with animals in public settings, 2005. National Association of State Public Health Veterinarians, Inc. (NASPHV). MMWR 2005;54 (No. RR-4):[1-20]

6. Centers for Disease Control and Prevention. Compendium of measures to prevent disease associated with animals in public settings, 2011. MMWR 2011;60(No. 4):[1-24].

7. Centers for Disease Control and Prevention. 2012. Notes from the Field: Escherichia coli O157:H7 Gastroenteritis Associated with a State Fair - North Carolina, 2011. MMWR 2012;60(51):1745-1746.

8. Centers for Disease Control and Prevention (CDC). 2010. Reptiles, amphibians and Salmonella. Available at: http:// www.cdc.gov/Features/SalmonellaFrogTurtle/ (accessed on 17 March 2011).

9. Chapman, Benjamin, Eversley, Tiffany, Fillion, Katie, MacLaurin, Tanya, and Powell, Douglas. 2010. Assessment of Food Safety Practices of Food Service Food Handlers (Risk Assessment Data): Testing a Communication Intervention (Evaluation of Tools). Journal of Food Protection, Vol. 73, No. 6, Pages 1101-1107.

10. Erdozain, G., Powell, D. bites. Petting Zoo Outbreaks. Available at: http:/ / bites.ksu.edu/petting-zoos-outbreaks (accessed on 12 August 2011).

11. Friedman CR, Torigian C, Shillam PJ, et al. 1998. An outbreak of salmonellosis among children attending a reptile exhibit at a zoo. J Pediatr 132:802--7. 
12. Goode B, O'Reilly C, Dunn J, Fullerton K, Smith S, Ghneim G, Keen J, Durso L, Davies M, Montgomery S. 2009. Outbreak of Escherichia coli 0157: H7 infections after Petting Zoo visits, North Carolina State Fair, October-November 2004. Arch Pediatr Adolesc Med. 2009 Jan;163(1):42-8.

13. Gould, D., et al., 2007. Measuring handwashing performance in health service audits and research studies. Journal of Hospital Infection, 66, 109-115.

14. Griffin, G. 2010. Review of the major outbreak of E. coli O157:H7 in Surrey, 2009. Report of the independent investigation committee June 2010.

Available at: http:// www.griffininvestigation.org.uk (accessed on 19 January 2011).

15. Health Protection Agency. Hand gels no substitute for handwashing on farm visits. 20 April 2011. Available at:

http://www.hpa.org.uk/webw/HPAweb\&HPAwebStandard/HPAweb_C/ 1296685526717? p=1287147958032_(accessed 5 September, 2011).

16. Marler, B. 2011. Outbreaks traced to state and county fairs, petting zoos, and community activities involving human-animal contact. Fair and Petting Zoo Safety. Available at: http:/ / www.fair-safety.com (accessed on 9 March 2011).

17. McMillan, M., Dunn, J.R., Keen, J.E., Brady, K.L., T.F. Jones. 2007. Risk behaviors for disease transmission among petting zoo attendees. J Am Vet Assoc 231(7):1036-8.

18. Moreno, M. 2009. Escherichia coli $\mathrm{O} 157$ and children. Advice for patients. Arch Pediatr Adolesc Med. 163 (1).

19. Pike-Paris, A. 2006. Environmental Health Topics: Petting Zoos. J Pediatr Nurs 32(2): 163-166.

20. Redway, K., Fawdar, S. 2008. A Comparative Study of Three Different Hand Drying Methods: Paper Towel, Warm Air Dryer, Jet Air Dryer. European Tissue Symposium.

21. Smith KE, Stenzel SA, Bender JB, et al. 2004. Outbreaks of enteric infections caused by multiple pathogens associated with calves at a farm day camp. Pediatr Infect Dis J 23:1098--104.

22. Stirling J., Griffith, M., Dooley, J. S., et al. 2007. Zoonoses associated with petting farms and open zoos. Vector-borne and Zoonotic 8:85-92.

23. Varma J. K., Greene K. D., Reller, M. E., et al. 2003. An outbreak of Escherichia coli $\mathrm{O} 157$ infection following exposure to a contaminated building. J Am Vet Med Assoc 290: 2709-2712.

24. Warshawsky, B., Gutmanis, I., Henry, B., Dow, J., Ahmed, R., Aldom, J., Alves, D., Chagla, A., Ciebin, B., Kolbe, F., Jamieson, F., F. Rodgers. 2002. 
Outbreak of Escherichia coli 0157:H7 related to animal contact at a petting zoo. Can J Infect Dis 13(3): 175-181.

25. Weese, S. J., McCarthy, L., Mossop, M., Martin, H., S. Lefebvre. 2007. Observation of practices at petting zoos and the potential impact on zoonotic disease transmission. Clin Infect Dis 45: 10-15.

26. Wilson, Sarah, Jacob, Casey J., Powell, Douglas. 2011. Behavior-change interventions to improve hand-hygiene practice: a review of alternatives to education. Critical Public Health Vol. 21, No. 1, 119-127.

27. World Health Organization, 2009. WHO Guidelines on Hand Hygiene in Health Care [online]. Available at: http://whqlibdoc.who.int/publications/2009/9789241597906_eng.pdf (accessed 22 January 2012).

28. Yamamoto, Y., Ugai, K., Takahashi, Y. 2005. Efficiency of Hand Drying for Removing Bacteria from Washed Hands: Comparison of Paper Towel Drying with Warm Air Drying. Infection Control and Hospital Epidemiology 26(3):316-20. 